\title{
A Study on Point Set Domination of Intuitionistic Fuzzy Graphs
}

\author{
${ }^{1} \mathrm{~S}$. Yahya Mohamed and ${ }^{2} \mathrm{R}$. Jahir Hussain, \\ ${ }^{1}$ P .G and Research Department of Mathematics, Govt. Arts College, Tiruchirappalli-620 022, India. \\ Email:yahya_md@yahoo.com. \\ ${ }^{2} P$.G and Research Department of Mathematics, Jamal Mohamed College, Tiruchirappalli-620 020, India. \\ Email:hssn_jhr@yahoo.com.
}

\begin{abstract}
Let $G=(V, E)$ be the IFG and $D$ be set of vertices, then $D$ is said to be Intuitionistic point set dominating set of $G$ if for every $S \subseteq V$ - D there exists a node $d \in D$ such that the Intuitionistic fuzzy sub graph $<S \cup\{d\}>$ induced by $S \cup\{d\}$ is a connected Intuitionistic fuzzy graph. In this paper we study some results on Intuitionistic Point set dominating set and its number of IFGs. Also we establish some theorems and bound of Intuitionistic psd-sets in purely semi-complete IFGs.
\end{abstract}

Keywords: Point set domination set of IFG, Set domination set of IFG, Semi complete IFG. 2010Mathematics Subject Classification: 03E72, 03F55, 05C69, $05 C 72$.

\section{Introduction:}

Atanassov [1] introduced the concept of intuitionistic fuzzy (IF) relations and intuitionistic fuzzy graphs (IFGs). Research on the theory of intuitionistic fuzzy sets (IFSs) has been witnessing an exponential growth in Mathematics and its applications. R. Parvathy and M.G. Karunambigai's paper [4] introduced the concept of IFG and analyzed its components. Nagoor Gani, A and Sajitha Begum, S [3] defined degree, Order and Size in intuitionistic fuzzy graphs and extend the properties. The concept of Domination in fuzzy graphs is introduced by A. Somasundaram and S. Somasundaram [10] in the year 1998. Parvathi and Thamizhendhi[5] introduced the concepts of domination number in Intuitionistic fuzzy graphs. Domination is active subject in fuzzy graphs and Intuitionistic fuzzy graphs, and has numerous applications to distributed computing, the web graph and adhoc networks. Point set domination number of a graph is introduced by E. Sampathkumar and L. Pushpa Latha [7] in 1993. Siva Rama Raju et. al. [9] analyzed the semi global domination in the crisp graph and semi-complete graph. In[11], we introduced semi complete IFG and Semi complementary IFG.

In this paper, we study some results on Intuitionistic fuzzy point set domination set and its number of IFGs and established the some bounds of the same. Also we analyzed Intuitionistic Psd-sets in purely semicomplete IFG and derived some theorems, which is useful to solve communication problems in more efficient way.

\section{Preliminary}

Definition 2.1: An Intuitionistic fuzzy graph is of the form $G=(V, E)$ where

(i) $\mathrm{V}=\{\mathrm{v} 1, \mathrm{v} 2, \ldots, \mathrm{Vn}\}$ such that $\mu_{1}: \mathrm{V} \rightarrow[0,1]$ and $\gamma_{1}: \mathrm{V} \rightarrow[0,1]$ denote the degree of membership and nonmembership of the element vi $\epsilon \mathrm{V}$, respectively, and $0 \leq \mu_{1}(\mathrm{vi})+\gamma_{1}$ (vi) $\leq 1$ for every vi $\epsilon \mathrm{V}$, $(\mathrm{i}=1,2, \ldots \ldots \mathrm{n})$,

(ii) $\mathrm{E} \subseteq \mathrm{V} \times \mathrm{V}$ where $\mu_{2}: \mathrm{Vx} \mathrm{V} \rightarrow[0,1]$ and $\gamma_{2}: \mathrm{V} \times \mathrm{V} \rightarrow[0,1]$ are such that $\mu_{2}(\mathrm{vi}, \mathrm{vj}) \leq \min \left[\mu_{1}(\mathrm{vi}), \mu_{1}(\mathrm{vj})\right]$ and $\gamma_{2}(\mathrm{vi}, \mathrm{vj}) \leq \max \left[\gamma_{1}(\mathrm{vi}), \gamma_{1}(\mathrm{vj})\right]$ and $0 \leq \mu_{2}(\mathrm{vi}, \mathrm{vj})+\gamma_{2}(\mathrm{vi}, \mathrm{vj}) \leq 1$ for every (vi, vj) $\epsilon$ E, $(\mathrm{i}, \mathrm{j}=1,2, \ldots, \mathrm{n})$

Definition 2.2: An IFG $H=<V^{\prime}, E^{\prime}>$ is said to be an Intuitionistic fuzzy sub graph (IFSG) of the IFG, $\mathrm{G}=<\mathrm{V}, \mathrm{E}>$ if $\mathrm{V}^{\prime} \subseteq \mathrm{V}$ and $\mathrm{E}^{\prime} \subseteq \mathrm{E}$. In other words, if $\mu_{1 \mathrm{i}}{ }^{\prime} \leq \mu_{1 \mathrm{i}} ; \gamma_{1 \mathrm{i}}{ }^{\prime} \geq \gamma_{1 \mathrm{i}}$ and $\mu_{2 \mathrm{ij}} \leq \mu_{2 \mathrm{ij}} ; \gamma_{2 \mathrm{ij}}{ }^{\prime} \geq \gamma_{2 \mathrm{ij}}$ for every $\mathrm{i}, \mathrm{j}=1,2 \ldots \ldots \ldots . . \mathrm{n}$.

The Induced Intuitionistic fuzzy sub graph defined as which is the Intuitionistic fuzzy sub graph of IFG $. \mu_{2}^{\prime}(v i, v j)=\min \left[\mu_{1}^{\prime}(v i), \mu_{1}^{\prime}(v j)\right]$ and $\gamma_{2}^{\prime}(v i, v j)=\max \left[\gamma_{1}^{\prime}(v i), \gamma_{1}^{\prime}(v j)\right]$

Definition 2.3: Let $G=(V, E)$ be a IFG. Then the cardinality of $G$ is defined as

$$
|\mathrm{G}|=\left|\sum_{\mathrm{vi} \in \mathrm{V}} \frac{1+\mu 1(\mathrm{vi})-\gamma 1(\mathrm{vi})}{2}+\sum_{\mathrm{vi}, \mathrm{vj} \in \mathrm{E}} \frac{1+\mu 2(\mathrm{vi}, \mathrm{vj})-\gamma 2(\mathrm{vi}, \mathrm{vj})}{2}\right|
$$

Definition 2.4: The vertex cardinality of IFG G is defined by $|\mathrm{V}|=\left|\sum_{\mathrm{vi} \in \mathrm{V}} \frac{1+\mu 1(\mathrm{vi})-\gamma 1(\mathrm{vi})}{2}\right|=\mathrm{p}$ and

The edge cardinality of IFG G is defined by $|\mathrm{E}|=\left|\sum_{\mathrm{vi}, \mathrm{vj} \in \mathrm{E}} \frac{1+\mu 2(\mathrm{vi}, \mathrm{vj})-\gamma 2(\mathrm{vi}, \mathrm{vj})}{2}\right|=\mathrm{q}$.

The vertex cardinality of IFG is called the order of $\mathrm{G}$ and denoted by $\mathrm{O}(\mathrm{G})$.

The edge cardinality of $\mathrm{G}$ is called the size of $\mathrm{G}$, denoted by $\mathrm{S}(\mathrm{G})$.

Definition 2.5: An edge $e=(x, y)$ of an $I F G G=(V, E)$ is called an effective edge if

$$
\mu_{2}(\mathrm{x}, \mathrm{y})=\mu_{1}(\mathrm{x}) \Lambda \mu_{1}(\mathrm{y}) \text { and } \gamma_{2}(\mathrm{x}, \mathrm{y})=\gamma_{1}(\mathrm{x}) \mathrm{V} \gamma_{1}(\mathrm{y}) \text {. }
$$


Definition 2.6: An Intuitionistic fuzzy graph is complete if $\mu_{2 \mathrm{ij}}=\min \left(\mu_{1 \mathrm{i}}, \mu_{1 \mathrm{j}}\right)$ and $\gamma_{2 \mathrm{ij}}=\max \left(\gamma_{2 \mathrm{i}}, \gamma_{2 \mathrm{j}}\right)$ for all $(\mathrm{vi}, \mathrm{vj}) \epsilon \mathrm{V}$.

Definition 2.7: An Intuitionistic fuzzy graph $G$ is said to be strong IFG if $\mu_{2}(\mathrm{x}, \mathrm{y})=\mu_{1}(\mathrm{x}) \Lambda \mu_{1}(\mathrm{y})$ and $\gamma_{2}(\mathrm{x}, \mathrm{y})=\gamma_{1}(\mathrm{x}) \mathrm{V} \gamma_{1}(\mathrm{y})$ for all $(\mathrm{vi}, \mathrm{vj}) \in \mathrm{E}$. That is every edge is effective edge.

Definition 2.8: The complement of an IFG G $=(\mathrm{V}, \mathrm{E})$ is denoted by $\bar{G}=(\bar{V}, \bar{E})$ and is defined as

i) $\bar{\mu}_{1}(v)=\mu_{1}(v)$ and $\bar{\gamma}_{1}(v)=\gamma_{1}(v)$

ii) $\bar{\mu}_{2}(\mathrm{u}, \mathrm{v})=\mu_{1}(\mathrm{u}) \Lambda \mu_{1}(\mathrm{v})-\mu_{2}(\mathrm{u}, \mathrm{v})$ and $\bar{\gamma}_{2}(\mathrm{u}, \mathrm{v})=\gamma_{1}(\mathrm{u}) \mathrm{V} \gamma_{1}(\mathrm{v})-\gamma_{2}(\mathrm{u}, \mathrm{v})$ for $\mathrm{u}, \mathrm{v}$ in $\mathrm{V}$ $\bar{G}$ also denoted by $\mathrm{G}^{\mathrm{c}}$.

Definition 2.9: Let $\mathrm{G}=(\mathrm{V}, \mathrm{E})$ be an IFG. The neighbourhood of any vertex $\mathrm{v}$ is defined as $\mathrm{N}(\mathrm{v})=\left(\mathrm{N}_{\mu}(\mathrm{v}), \mathrm{N}_{\gamma}(\mathrm{v})\right)$, Where $\mathrm{N}_{\mu}(\mathrm{v})=\left\{w \in V ; \mu_{2}(v \cdot w)=\mu_{1}(v) \Lambda \mu_{1}(\mathrm{w})\right\}$ and $\mathrm{N}_{\gamma}(\mathrm{v})=\left\{w \in V ; \gamma_{2}(v \cdot w)=\gamma_{1}(v) V \gamma_{1}(w)\right\} . \quad \mathrm{N}[\mathrm{v}]=\mathrm{N}(\mathrm{v}) \cup\{\mathrm{v}\}$ is called the closed neighbourhood of $\mathrm{v}$.

Definition 2.10: The neighbourhood degree of a vertex is defined as $d_{N}(v)=\left(d_{N \mu}(v), d_{N \gamma}(v)\right)$ where $\mathrm{d}_{\mathrm{Nu}}(\mathrm{v})=\sum_{w \in N(v)} \mu_{1}(w)$ and $\mathrm{d}_{\mathrm{Ny}}(\mathrm{v})=\sum_{w \in N(v)} \gamma_{1}(w)$.

The minimum neighbourhood degree is defined as $\delta_{\mathrm{N}}(\mathrm{G})=\left(\delta_{\mathrm{N} \mu}(\mathrm{v}), \delta_{\mathrm{N} \gamma}(\mathrm{v})\right)$, where $\delta_{\mathrm{N} \mu}(\mathrm{v})=\Lambda\left\{\mathrm{d}_{\mathrm{N} \mu}(\mathrm{v})\right.$ : $\mathrm{v} \in \mathrm{V}\}$ and $\delta_{\mathrm{N \gamma}}(\mathrm{v})=\Lambda\left\{\mathrm{d}_{\mathrm{N} \gamma}(\mathrm{v}): \mathrm{v} \in \mathrm{V}\right\}$.

Definition 2.11: The effective degree of a vertex $v$ in a IFG G $=(V, E)$ is defined to be sum of the effective edges incident at $\mathrm{v}$, and denoted by $\mathrm{d}_{\mathrm{E}}(\mathrm{v})$. The minimum effective degree of $\mathrm{G}$ is $\delta_{\mathrm{E}}(\mathrm{G})=\Lambda\left\{\mathrm{d}_{\mathrm{E}}(\mathrm{v}) / \mathrm{v} C \mathrm{~V}\right\}$

Definition 2.12: Let $G=(V, E)$ be an IFG. Let $u, v \in V$, we say that $u$ dominated $v$ in $G$ if there exist a strong arc between them. A subset $\mathrm{D} \subseteq \mathrm{V}$ is said to be dominating set in $\mathrm{G}$ if for every $\mathrm{v} \in \mathrm{V}$-D, there exist $\mathrm{u}$ in $\mathrm{D}$ such that $\mathrm{u}$ dominated $\mathrm{v}$. The minimum scalar cardinality taken over all dominating set is called domination number and is denoted by $\gamma(\mathrm{G})$. The maximum scalar cardinality of a minimal domination set is called upper domination number and is denoted by the symbol $\Gamma(\mathrm{G})$.

Definition 2.13: Let $G=(V, E)$ be an IFG. A subset $D \subseteq V$ is said to be total dominating set in $G$ if every vertex in $\mathrm{V}$ is dominated by a node in $\mathrm{D}$. The minimum cardinality of all total dominating sets is called total Intuitionistic fuzzy domination number and denoted by $\gamma_{t}(\mathrm{G})$

Definition 2.14: An independent set of an Intuitionistic fuzzy graph $G=(V, E)$ is a subset $S$ of $V$ such that no two vertices of $\mathrm{S}$ are adjacent in $\mathrm{G}$.

Definition 2.15: A Bipartite IFG, $G=(V, E)$ is said to be complete Bipartite IFG, if $\mu_{2}\left(v_{i}, v_{j}\right)=\mu_{1}\left(v_{i}\right) \Lambda \mu_{1}\left(v_{j}\right)$ and $\gamma_{2}\left(\mathrm{v}_{\mathrm{i}}, \mathrm{v}_{\mathrm{j}}\right)=\gamma_{1}\left(\mathrm{v}_{\mathrm{i}}\right) \mathrm{V} \gamma_{1}\left(\mathrm{v}_{\mathrm{j}}\right)$ for all $\mathrm{v}_{\mathrm{i}} \in \mathrm{V}_{1}$ and $\mathrm{v}_{\mathrm{j}} \in \mathrm{V}_{2}$. It is denoted by $K_{V 1 i, V 2 i}$.

Definition 2.16: $G=(V, E)$ be a connected IFG with effective edges which is said to be semi complete IFG, if every pair vertices have a common neighbor in $\mathrm{G}$.

An IFG, $G$ is said to be purely semi complete IFG, if and only if $G$ is semi complete IFG but not complete IFG.

\section{Intuitionistic Point set Domination of IFG}

Definition 3.1: Let $G=(V, E)$ be IFG. A set $D \subseteq V$ is said to be Intuitionistic fuzzy Point set dominating set of $\mathrm{G}$ if for every $\mathrm{S} \subseteq \mathrm{V}-\mathrm{D}$ there exists a node $\mathrm{d} \in \mathrm{D}$ such that the Intuitionistic fuzzy sub graph $<\mathrm{S} \cup\{\mathrm{d}\}>$ induced by $\mathrm{SU}\{\mathrm{d}\}$ is a connected strong Intuitionistic fuzzy graph.

The Intuitionistic point set fuzzy domination number $\gamma_{\mathrm{ip}}(\mathrm{G})$ of $\mathrm{G}$ is the minimum scalar cardinality of Intuitionistic fuzzy Point set dominating sets.

\section{Example 3.2:}

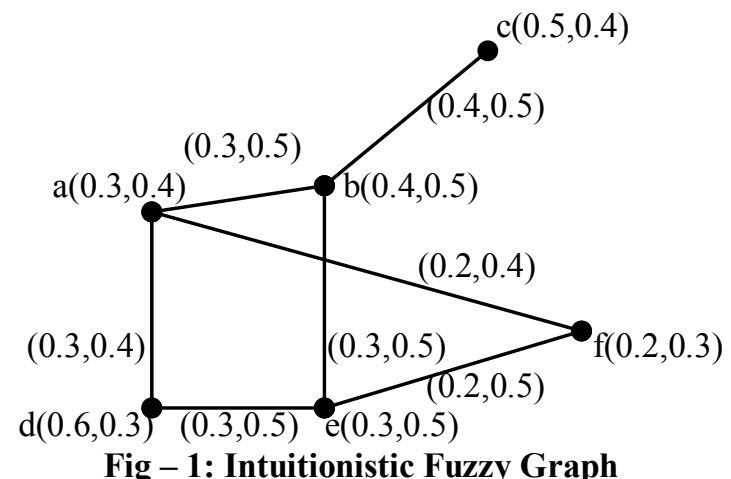

Here $|\mathrm{a}|=0.45,|\mathrm{~b}|=0.45,|\mathrm{c}|=0.55,|\mathrm{~d}|=0.65,|\mathrm{e}|=0.4,|\mathrm{f}|=0.45$ and $\gamma_{\mathrm{ip}}$ - set is $\{\mathrm{c}, \mathrm{d}, \mathrm{f}\}$. Then $\gamma_{\mathrm{ip}}(\mathrm{G})=1.65$. 
Theorem: 3.3: For any Intuitionistic fuzzy graph $\mathrm{G}, \operatorname{Min}\{|\mathrm{vi}|\} \leq \gamma_{\mathrm{ip}}(\mathrm{G})<\mathrm{p}$

Proof: If $\mathrm{G}$ is complete Intuitionistic fuzzy graph then $\gamma_{\mathrm{ip}}(\mathrm{G})$-set contains only one vertex and we know that $\gamma_{\text {ip }}(\mathrm{G})-$ set have at least one vertex. That is $\operatorname{Min}\{|\mathrm{vi}|\} \leq \gamma_{\mathrm{ip}}(\mathrm{G})$

Also, Suppose $\mathrm{S}$ be the Intuitionistic fuzzy point set dominating set and $\mathrm{V}-\mathrm{S}$ is not an empty set then $\mathrm{S}$ contain at most $n-1$ vertices. That is the cardinality of $S$ is less than $p$. Therefore $\gamma_{i p}(G)<p$ Hence we get $\operatorname{Min}\{\mid$ vi $\mid\} \leq \gamma_{\text {ip }}(\mathrm{G})<\mathrm{p}$.

\section{Remark 3.4:}

(i) $\gamma_{\mathrm{ip}}\left(\mathrm{K}_{\mathrm{n}}\right)=\operatorname{Min}\left\{\left|\mathrm{v}_{\mathrm{i}}\right|\right\}$ where $\mathrm{v}_{\mathrm{i}} \in \mathrm{V}$

(ii) $\gamma_{\mathrm{ip}}\left(\mathrm{K}_{\mathrm{vl}, \mathrm{vj}}\right)=\left|\mathrm{v}_{1}\right|$ where $\mathrm{v}_{1}$ is centre vertex of IFG and $\mathrm{j}=1,2, . ., \mathrm{n}$

(iii) $\gamma_{\text {ip }}\left(K_{V 1 i, V 2 j}\right)=\operatorname{Min}\left\{\left|\mathrm{v}_{1 \mathrm{i}}\right|+\mid \mathrm{v}_{2 \mathrm{j}}\right\}$ where $\mathrm{v}_{\mathrm{i}} \in \mathrm{V}_{1}$ and $\mathrm{v}_{\mathrm{j}} \in \mathrm{V}_{2}$

(iv) If $\mathrm{Cn}$ is Intuitionistic fuzzy graph and the induced crisp graph is cyclic graph with $\mathrm{n}$ vertices then $\gamma_{\mathrm{ip}}(\mathrm{Cn})=\sum_{j=1}^{n-2} v_{j} \quad$ where $\mathrm{v}_{\mathrm{j}}=\operatorname{Min}\left\{\left|\mathrm{v}_{\mathrm{i}}\right|\right\}, \mathrm{i}=1,2, \ldots, \mathrm{n}$.

Theorem 3.5: Every Intuitionistic fuzzy point set dominating set is Intuitionistic fuzzy dominating set but not conversely.

Proof: Let $\mathrm{S}$ be Intuitionistic fuzzy psd-set, By the definition it is always a dominating set of $\mathrm{G}$ but may not minimum. But the for the minimal dominating set $\mathrm{D}$ there exist a vertex $\mathrm{d} \in \mathrm{D},<\mathrm{S} \cup\{\mathrm{d}\}>$ may not be connected where $\mathrm{S} \subseteq \mathrm{V}-\mathrm{D}$. That is converse need not true.

Example: In fig $-1, \gamma$-set is $D=\{a, b\}$ and $\gamma(G)=0.9$. Also $\gamma_{\text {ip }}$ - set is $\{c, d, f\}$.

Here, $\gamma$-set is not $\gamma_{\mathrm{ip}}(\mathrm{G})$-set. since, for the set $\mathrm{S}=\{\mathrm{c}, \mathrm{f}\} \subseteq \mathrm{V}$ - D, both $<\mathrm{S} \cup\{\mathrm{a}\}>$ and $<\mathrm{S} \cup\{\mathrm{b}\}>$ are disconnected. Also $\gamma(\mathrm{G}) \leq \gamma_{\mathrm{ip}}(\mathrm{G})$

Theorem 3.6: Let $G=(V, E)$ be the IFG and $D$ the Intuitionistic fuzzy psd-set of $G$. Then $<V-D>$ is a proper sub graph of a component $\mathrm{H}$ of $\mathrm{G}$.

Proof: Suppose there exist vertices $u$ and $v$ belonging to two different components of $G$.

Since $\mathrm{D}$ is a Intuitionistic fuzzy psd-set of $\mathrm{G}$, there much exist $\mathrm{w} \in \mathrm{D}$ such that $<\{\mathrm{u}, \mathrm{v}, \mathrm{w}\}>$ is stongly connected IFG.

Which is contradiction to our assumption.

i.e) $\mathrm{V}-\mathrm{D} \subseteq \mathrm{V}(\mathrm{H})$ for some component $\mathrm{H}$ of $\mathrm{G}$. Further $\mathrm{D} \cap \mathrm{V}(\mathrm{H}) \neq \emptyset$ which implied $<\mathrm{V}-\mathrm{D}>$ is a proper sub graph of $\mathrm{H}$. Hence the proof.

Theorem 3.7: Let $\mathrm{G}=(\mathrm{V}, \mathrm{E})$ be a strongly connected IFG and $\mathrm{S}$ is the Intuitionistic fuzzy dominating set of $\mathrm{G}$. If for every $\mathrm{D} \subseteq \mathrm{V}-\mathrm{S}$ and $<\mathrm{D}>$ has a Intuitionistic fuzzy dominating set itself, then $\mathrm{S}$ is Intuitionistic fuzzy psdset of G.

Proof: By the given hypothesis, $\angle D>$ is connected for every $D \subseteq V-S$. Since $S$ is dominating set of $G$ then there is a $u \in S$ such that $u$ is adjacent with some vertex of $D$.

Hence $<\mathrm{DU}\{\mathrm{u}\}>$ is connected. Thus $\mathrm{S}$ is a Intuitionistic fuzzy psd-set of G.

Theorem 3.8: Let $G=(V, E)$ be IFG. If $S$ is Intuitionistic fuzzy Point set dominating set and $u, v \in V-S$ then between $u$ and $v$ there are at most two strong arcs.

Proof: Let $\mathrm{D}=\{\mathrm{u}, \mathrm{v}\}$.

Let $\mathrm{u}$ and $\mathrm{v}$ are not adjacent and $\mathrm{S}$ is the Intuitionistic fuzzy psd-set, Then there exist a vertex $\mathrm{x}$ in $\mathrm{S}$ such that the sub graph $<\{\mathrm{u}, \mathrm{v}, \mathrm{x}\}>$ is Strongly connected.

This implies between the vertices $u$ and $v$ there are at most two strong edges only.

Theorem 3.9: Let $G=(V, E)$ be the purely semi complete $I F G$ and $S$ is a Intuitionistic fuzzy dominating set in $\mathrm{G}$, then $\mathrm{S}$ is Intuitionistic psd-set if and only if for any independent vertex set $\mathrm{B} \subseteq \mathrm{V}$ - $\mathrm{S}$. there is a $\mathrm{v} \in \mathrm{S}$ such that every vertex of $\mathrm{B}$ is strongly adjacent to $\mathrm{v}$ in $\mathrm{G}$.

Proof: Given $\mathrm{S}$ is a Intuitionistic psd-set in $\mathrm{G}$ and $\mathrm{B}$ be any independent vertex set $\mathrm{B} \subseteq \mathrm{V}-\mathrm{S}$, then there is a $\mathrm{V}$ $\in \mathrm{S}$ such that $<\mathrm{S} \cup\{\mathrm{v}\}>$ is strongly connected. Since no two elements in $\mathrm{B}$ are adjacent then by previous theorem between two vertices there are two strong arcs. Therefore $\mathrm{G}$ is purely semi complete IFG and $\mathrm{S}$ is a Intuitionistic fuzzy dominating set in $\mathrm{G}$.

Conversely, suppose $\mathrm{S}$ is a Intuitionistic fuzzy dominating set in $\mathrm{G}$ and any independent vertex set $\mathrm{B} \subseteq \mathrm{V}-\mathrm{S}$,. there is a $\mathrm{v} \in \mathrm{S}$ such that every vertex of $\mathrm{B}$ is strongly adjacent to $\mathrm{v}$ in $\mathrm{G}$.

Let $\mathrm{D} \subseteq \mathrm{V}-\mathrm{S}$ and $<\mathrm{D}>$ is connected then $\mathrm{S}$ is Intuitionistic psd-set. Otherwise let there are more than one components of $\mathrm{D}$ then $\mathrm{B}=\{\mathrm{v} 1, \mathrm{v} 2, \ldots, \mathrm{vn}\}$ is an independent set in $\mathrm{G}$. 
Now by hypothesis, Since each vi is adjacent to $v$ in $G$ then there exist $v \in S$ such that $\langle\mathrm{B} \cup\{v\}\rangle$ is connected. Therefore, $<\mathrm{D} \cup\{\mathrm{v}\}>$ is connected.

Hence, $\mathrm{S}$ is Intuitionistic psd-set in $\mathrm{G}$.

Theorem 3.10: Let $G=(V, E)$ be the purely semi complete IFG and has a unique path between any pair of vertices in $\mathrm{G}$, then the intersection all Intuitionistic psd-sets in $\mathrm{G}$ is a singleton set.

Proof: Since G is purely semi complete IFG then G is a union of edge disjoint triangles having a common vertex, say u.

It follows that any Intuitionistic psd-set contains the vertex u. Also $\{u\}$ itself is Intuitionistic psd-set. Hence their intersection is $\{u\}$.

Proposition 3.11 : The converse of the above theorem is false. i.e) the intersection all Intuitionistic psd-sets in $\mathrm{G}$ is a singleton set but $\mathrm{G}$ need not be purely semi complete IFG with unique path between any pair of vertices in G.

Example 3.12:

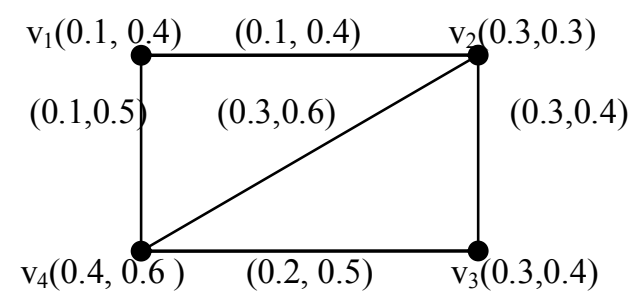

Fig - 2: Purely Semi-Complete IFG

Here, for the vertices $\left\{\mathrm{v}_{2}, \mathrm{v}_{4}\right\}$ there are two different paths.

Theorem 3.13: Let $G=(V, E)$ be a strongly connected IFG and $S$ is the Intuitionistic fuzzy dominating set of $G$ which is not Intuitionistic fuzzy psd-set in $\mathrm{G}$ then the minimal $\gamma$-set contains at least two vertices.

Proof: Since $\mathrm{S}$ is not a Intuitionistic fuzzy psd-set then there is a D $\subseteq \mathrm{V}-\mathrm{S}$ such that $<\mathrm{D} \cup\{\mathrm{v}\}\rangle$ is not connected for any $\mathrm{v} \in \mathrm{S}$. Hence $<\mathrm{D}>$ is not connected and therefore has at least two components.

So at least two vertices in $\mathrm{S}$ are not dominated by a single vertex from $\mathrm{S}$. Which implies $\gamma$-set contains at least two vertices.

Remark 3.14: (i) In Fig - $1, \gamma$-set is $\{a, b\}$ which is not Intuitionistic fuzzy psd-set. $\gamma$-set has two vertices.

(ii) The Converse is not true. That is, $\mathrm{S}$ is $\gamma$-set which contains two vertices then $\mathrm{S}$ is also $\gamma_{\mathrm{ip}}$-set may happen.

Definition 3.15: Let $G=(V, E)$ be IFG. A set $D \subseteq V$ is said to be Intuitionistic fuzzy set-domination set if every set $\mathrm{T} \subseteq \mathrm{V}$-D, there exists a non empty set $\mathrm{S} \subseteq \mathrm{D}$ such that the induced sub graph $<\mathrm{S} \cup \mathrm{T}>$ is connected.

The minimum cardinality taken over all Intuitionistic fuzzy set - domination sets is called Intuitionistic fuzzy set domination number and denoted by $\gamma_{\mathrm{s}}(\mathrm{G})$

Theorem 3.16 : Let $\mathrm{G}=(\mathrm{V}, \mathrm{E})$ be IFG then, $\gamma(\mathrm{G}) \leq \gamma_{\mathrm{s}}(\mathrm{G}) \leq \gamma_{\mathrm{ip}}(\mathrm{G})$.

Proof: Let $\mathrm{D}$ be a Intuitionistic fuzzy Point set dominating set of IFG, for every set $\mathrm{T} \subseteq \mathrm{V}$-D there exist a non empty singleton set $\{\mathrm{v}\} \in \mathrm{S} \subseteq \mathrm{D}$ such that the sub graph $<\mathrm{S} \cup \mathrm{T}>$ is connected. Thus $\mathrm{D}$ is a Intuitionistic fuzzy set -domination set. Then $\gamma_{\mathrm{s}}(\mathrm{G}) \leq \gamma_{\mathrm{ip}}(\mathrm{G})$.

Suppose D be a Intuitionistic fuzzy set -domination set of IFG, the for every set $\{x\}=T \subseteq V$-D there exist a non empty singleton set $\{\mathrm{v}\} \in \mathrm{S} \subseteq \mathrm{D}$ such that the sub graph $\langle\mathrm{SU} \mathrm{T}>$ is connected. Thus $\mathrm{D}$ is a Intuitionistic fuzzy domination set. Then $\gamma(\mathrm{G}) \leq \gamma_{\mathrm{s}}(\mathrm{G})$

Therefore we have $\gamma(\mathrm{G}) \leq \gamma_{\mathrm{s}}(\mathrm{G}) \leq \gamma_{\mathrm{ip}}(\mathrm{G})$.

Remark: The converses are need not be true.

\section{Conclusion}

Here, we discussed some results on Intuitionistic fuzzy points set domination and set domination on IFGs and derived some bounds in the standards IFGs and semi complete IFG. Also we established some theorems on Intuitionistic psd-sets in purely semi complete IFG which is useful to solve communication network, medical diagnosis problems and Transportation networks. Further we are going to analyze Intuitionistic set domination in Semi complete IFG and compare with other dominating parameters. 


\section{References}

[1]. Atanassov KT. Intuitionistic fuzzy sets: theory and applications. Physica, New York, 1999.

[2]. Harary,F., Graph Theory, Addition Wesley, Third Printing, October 1972.

[3]. Nagoor Gani. A and Shajitha Begum.S, Degree, Order and Size in Intuitionistic Fuzzy Graphs, International Journal of Algorithms, Computing and Mathematics,(3)3 (2010).

[4]. Parvathi, R. and Karunambigai, M.G., Intuitionistic Fuzzy Graphs, Computational Intelligence, Theory and applications, International Conference in Germany, Sept 18 -20, 2006

[5]. Parvathi,R., and Thamizhendhi, G. Domination in Intuitionistic fuzzy graphs, Fourteenth Int. conf. on IFGs, Sofia, NIFS Vol.16, 2, 39-49, 15-16 May 2010.

[6]. Kulli,V.R., Theory of domination in graph, Vishwa International Publications, 2010

[7]. Sampathkumar. E., and Pushpa Latha. L., Point set domination number of a Graph, Indian J. pure appl. Math.,24(4); 225-229, April 1993 .

[8]. Sampathkumar.E, and Walikar.H.B., The connected domination number of a graph, J. math. Phys. Sci., 13 (1979), $607-613$.

[9]. Siva Rama Raju.S.V., and Kumar Addagarla. R., Semi Global Domination, International Journal of Mathematical Archieve-3(7), 2012, 2589-2593.

[10]. Somasundaram, A and Somasundaram, S., Domination in Fuzzy graph-I, Patter Recognition Letter 19(9), 1998, 787-791

[11]. Yahya Mohamed. S., and Jahir Hussain. R., Semi global dominating set of Intuitionistic fuzzy graphs, IOSR-JM, Vol. 10, Issue 4, Ver. II (Jul-Aug2014), pp 23-27.

[12]. Zimmermann, H.J., Fuzzy set theory and its Applications, Kluwer-Nijhoff, Boston, 1985.

[13]. B.D. Acharya and P. Gupta, On point-set dominatiuon in graphs, In: Proceedings of National Seminar on Recent Development in Mathematics, Karnatak University, Dharwad, (1996), 106-108. 